\title{
Analysis of Medical School Alumni's Perception on the Importance and Educational Needs of Outcomes
}

\section{Su-Jin Chae}

\author{
Brain Korea 21 Project, Ajou University School of Medicine, Suwon, Korea
}

\section{의학교육 학습성과에 대한 의과대학 졸업생의 중요도 및 교육요구도 인식 조사}

아주대학교 의과대학 BK21 사업단

\section{채수진}

Purpose: This study examined how important graduates consider the 15 items of educational outcomes (importance) and how much they feel that they have achieved at graduation (accomplishment), determined whether outcomes varied according to profession, and assessed what graduates' educational needs are.

Methods: A questionnaire was given to 85 graduates of Ajou University School of Medicine. The data were analyzed by Kruskal-Wallis test using SPSS 12.0. The Borich formula was used to examine graduates' educational needs.

Results: Educational outcomes, such as medical knowledge and clinical skills at the basic medical levels, communication skills, and social accountabilities, were considered important in every profession. The Kruskal-Wallis test results demonstrated that the 15 items of educational outcomes were not significantly different between careers $(p>0.05)$, but the average scores were. Doctors in private practice placed an emphasis on keeping a healthy body and mind and continuing lifelong learning; doctors in general hospitals emphasized understanding the health care industry's policies; and professors at university hospitals focused on proper medical ethics - the house staff emphasized teamwork with colleagues.

Conclusion: These test results can be used as basic materials for reflecting on educational objectives, student performance, and revision of the curricula. Further studies on how to measure educational outcomes are necessary.

Key Words: Outcome, Competence, Outcome-based education, Educational needs

\section{서론}

최근의 사회적 변화에 따른 교육개혁 흐름 가운데 주목할
만한 것은 ‘학습성과(outcome)'에 관한 관심이 고조되고 있다 는 점이다. 종래의 학교교육이 지식 전달에 기반한 교육이라 고 한다면 이러한 지식 전달 중심의 교육은 학생들을 최근의 변화하는 환경에 적절히 적응시키는 데 한계가 있다. 따라서
Received: February 23, 2010 - Revised: April 28, $2010 \bullet$ Accepted: May 10, 2010 Corresponding Author: Su-Jin Chae

BK21, Ajou University School of Medicine, San 5 Wonchun-dong Yeongtong-gu, Suwon 443-721, Korea

TEL) 031-219-4465 FAX) 031-219-4093 E-mail) edujin@ajou.ac.kr

* This research was presented at the 25th Korean Medical Education Conference in 2009.
Korean J Med Educ 2010 Jun; 22(2): 113-120. doi: $10.3946 / \mathrm{kjme} .2010 .22 .2 .113$.

pISSN: 2005-727X elSSN: 2005-7288

(C) The Korean Society of Medical Education. All rights reserved. 
학교교육의 목적을 지식 전달이 아닌 결과에 초점을 두어야 한다는 논의와 함께 학습성과에 대한 관심이 증가하고 있다 $[1,2,3]$.

의학교육도 예외가 아니다. 1990년대 후반부터 영국의 Dundee 의과대학, 미국의 Brown 의과대학, Mayo 의과대학, 파키스탄의 Aga Khan 의과대학, 싱가포르의 Yong Loo Lin 의과대학 등 세계 몇몇 의과대학이 학습성과바탕교육(outcome based education)이라는 개념을 받아들이고 있으며 [4,5], 미국의과대학협회(Association of American Medical College), 캐나다 의사협회(Royal College of Physicians and Surgeons of Canada), 졸업 후 의학교육 인증평가원 (Accreditation Council for Graduate Medical Eduation, ACGME), 영국의학협의회(General Medical Council, GMC) 등 의학교육 학회나 협회들도 학습성과를 개발하고 일선 현 장에 전달하는 데에 노력을 경주하고 있다[6].

학습성과란, 의학교육 프로그램을 이수한 결과로 학생들이 졸업 당시 갖추고 있음을 입증하여 주기를 바라는 일종의 졸 업 역량이다[7,8]. 다시 말하면, 학습성과는 각 의과대학의 역 사와 전통, 구성원의 차이와 관계없이 의학 분야를 전공한 학 생이라면 분명히 졸업 전에 학교에서 배우고 나와야 하는 최 소한의 능력과 자질을 의미한다[9]. 이러한 학습성과는 단순 히 아는 수준을 넘어서 학습자가 그들이 아는 것을 가지고 실 제로 '무엇을 할 수 있는지'에 초점이 있다. 학습성과바탕교육 과정(outcome based curriculum)은 좋은 의사의 정의(definition)에서 시작한다. 전통적인 교육과정(flexnerian curriculum)이 먼저 의학의 중요한 지식을 선정하고 그 지식을 가 르치는 절차를 따른다고 한다면, 학습성과바탕교육과정은 성 공적인 졸업생의 능력을 먼저 정의하고 그 능력을 졸업 당시 달성하였는가를 측정하는 전통적인 교육과정과는 반대 방향 의 절차를 따른다[10].

세계적으로 학습성과바탕교육에 대한 관심과 논의가 증대 되고 있으며 그 필요성이 제기되고 있으나 아직까지 국내 의 학교육에서는 본격적인 탐색이나 연구가 그리 많지 않다. 학 습성과가 구체적으로 무엇을 의미하며, 대학에 어떻게 적용 을 해야 하는지, 그리고 학습성과바탕교육과정이 우리나라 의과대학에서 그 실천이 가능하며, 어떠한 한계가 있는지 등 의 연구가 이루어져야 한다.
본 연구는 국내 의과대학에서 공통적으로 선정할 만한 학 습성과가 무엇인가라는 물음에서부터 출발한다. 먼저, 아주대 학교 의과대학 졸업생을 대상으로 졸업 후 의료 현장에서 활 동하면서 중요하다고 생각되는 의사의 자질과 능력에 대한 중요도를 조사하고, 그러한 자질이 직업에 따라 차이가 있는 지를 살펴볼 것이다. 다음으로, 학습성과에 대한 중요도 인식 수준과 현재 자신의 능력 수준 간의 차이를 조사하여 의학교 육에 바라는 졸업생들의 교육 요구도를 알아보고자 한다.

\section{대상 및 방법}

\section{1. 조사 대상}

본 연구는 2009년 동문의 날에 참석한 졸업생 120 명을 대 상으로 설문조사를 실시하였다. 설문에 응답한 졸업생은 120 명 중 85명으로 $71 \%$ 의 응답률을 보였다. 85 명 응답자들의 직 업은 개업의 $13 \%$ (11명), 봉직의 $11 \%$ (9명), 대학교원은 $33 \%$ (28명), 전공의는 39\% (33명), 기타(군복무자, 제약회사)가 $5 \%$ (4명) 등의 분포를 나타내었다(Table 1).

\section{2. 조사 방법}

먼저 학습성과를 선정하기 위해서 41 개 의과대학 및 의학 전문대학원의 교육목표를 조사하여 질적 분석을 실시하였다. 1 단계는 학장협의회가 발간하는 의과대학 교육현황집(2004 년 2005년)과 인터넷에 공개된 의과대학 및 의학전문대학원 의 교육목표 190개를 목록으로 작성한 뒤, 유사한 내용을 범 주화하여 학습성과 25 개를 추출하였다. 의학교육 학습성과를

Table 1. Analysis of Subjects by Profession

\begin{tabular}{lcc}
\hline \multicolumn{1}{c}{ Profession } & Frequency & Percentage \\
\hline Private practice & 11 & 12.9 \\
General hospital & 9 & 10.6 \\
University hospital & 28 & 32.9 \\
House staff & 33 & 38.8 \\
Others & 4 & 4.7 \\
Total & 85 & 100 \\
\hline
\end{tabular}


Table 2. Fifteen Outcomes of Medical Education

1. Medical knowledge and clinical skills at the primary physician level

2. An ability to research critically

3. An ability to solve problems

4. An ability to speak foreign languages

5. An ability to demonstrate accountability to patients, society, and the profession

6. A knowledge of contemporary issues in society, economy, environment, and law

7. A critical understanding of ethical and moral responsibilities

8. An ability to function on teams (peers)

9. An ability to keep a health body and mind

10. An ability to communicate effectively with patients and families

11. An ability to gather essential information and use up-to-date techniques

12. A recognition of needs and an ability to engage in life-long learning

13. An ability to engage in international collaboration and medical activity

14. Self-development and self-assessment

15. An ability to assume leadership roles

유의미한 것으로 인식하기 위해서 CanMed 2000 Project [11]에서 제안한 21세기 의사가 갖추어야 할 역량에 대한 21 개 항목과 Lee \& Ahn [12]이 연구한 '좋은 의사' 특성 33개 항목을 활용하여 17 개의 항목을 추출하였다. 2 단계로 연구자 가 추출한 17 개의 항목을 의과대학 교수 2 인과 교육학자 1 인 이 내용과 문구를 검토하였다. 검토 결과 유사한 내용으로 판 단된 2 개의 항목을 제외하고 15 개의 학습성과를 최종적으로 선정하고(Table 2) 설문지를 작성하였다. 설문지는 졸업 후 의료현장에서 활동하면서 자신이 중요하다고 생각하는 의사 의 자질 및 능력(중요도)과 이러한 자질과 능력을 졸업한 현 재 어느 정도 성취하였다고 생각하는지(성취도)를 함께 표시 하도록 구성하였다. 설문도구의 신뢰도는 Cronbach alpha 0.947 이었다.

\section{3. 분석 방법}

수집된 자료는 SPSS 12.0 통계 프로그램(SPSS Inc., Chicago, USA)을 사용하였다. 졸업생들의 직업에 따라 학습 성과에 대한 중요성 인식 정도에 차이가 있는지를 알아보기
위해서 Kruskal-Wallis 비모수검정법을 실시하였으며, 교육 요구도는 Borich 공식을 사용하여 값을 구하였다[13].

$$
\text { 교육요구도 }=\frac{\left\{\sum(\mathrm{RCL}-\mathrm{PCL})\right\} \times \underline{\mathrm{RCL}}}{\mathrm{N}}
$$

$\mathrm{RCL}$ : 내가 생각하는 중요성

$\mathrm{RCL}$ : 내가 생각하는 중요성의 평균

$\mathrm{PCL}$ : 내가 알고 있는 수준

$\mathrm{N}$ : 사례수

교육 요구도 공식에서 RCL은 졸업생들이 생각하는 학습성 과의 중요성 점수를 의미하고, PCL은 졸업생이 생각하는 현 재 자신의 능력수준 점수를 의미한다. RCL은 각 학습성과에 대하여 전체 졸업생이 생각하고 요구하는 중요성 점수의 평 균값이다. 교육 요구도는 각 사례마다 두 수준의 차이를 모두 합산한 값 \{ \}에 중요하다고 생각하는 중요도 점수의 평균값 을 곱한 다음, 전체 사례수로 나눈 값을 말한다. 교육 요구도 분석은 요구되는 능력수준과 현재 자신의 능력 수준의 차이 를 각 사례마다 모두 합산하기 때문에 결과 값의 범위가 넓어 지고 항목들 간 변별이 용이하다는 장점이 있다.

\section{결과}

\section{1. 졸업생들이 생각하는 학습성과의 중요도 및 현 재 능력수준 정도}

Table 3은 졸업생들이 인식하는 학습성과의 중요도와 졸업 생 자신이 생각하는 현재 능력 수준 정도를 나타낸 것이다. 먼 저 학습성과의 중요도 점수를 보면, 5 점을 기준으로 할 때 4 점대의 학습성과는 '환자와 환자 가족과 효율적으로 의사소통 하는 능력'(4.33), '일차 의료수준의 의학적 지식과 술기' (4.30), '의사로서의 사회적 책임감'(4.15), '올바른 의학윤리 관(4.11), '건강한 정신과 신체 유지'(4.02), '동료와의 협동정 신'(4.00) 등이었으며, 3점대는 '의학활동참여 능력'(3.38), '의 학연구 수행 능력'(3.49), '문제해결력'(3.69), '외국어'(3.79), '교양지식'(3.67), '정보수집'(3.57), '자기평가'(3.85), '리더십' 
Table 3. Alumni's Perception of Outcomes' Importance and Self Ability

\begin{tabular}{lll}
\hline \multicolumn{1}{c}{ Outcomes } & Importance & Self ability \\
\cline { 2 - 3 } & Mean (SD) & Mean (SD) \\
\hline 1. Medical knowledge and clinical skills & $4.30(0.80)$ & $3.49(0.80)$ \\
2. Research & $3.49(0.94)$ & $2.95(0.77)$ \\
3. Solving problems & $3.69(0.86)$ & $2.80(0.82)$ \\
4. Foreign languages & $3.79(0.90)$ & $2.76(0.91)$ \\
5. Accountability & $4.15(0.73)$ & $3.40(0.72)$ \\
6. Knowledge of contemporary issues & $3.67(0.89)$ & $3.05(0.93)$ \\
7. Ethics and morals & $4.11(0.77)$ & $3.59(0.90)$ \\
8. Teamwork & $4.00(0.74)$ & $3.55(0.85)$ \\
9. Keep a health body and mind & $4.02(0.96)$ & $3.26(0.83)$ \\
10. Communication & $4.33(0.77)$ & $3.32(0.78)$ \\
11. Information management & $3.57(0.87)$ & $2.79(0.85)$ \\
12. Life-long learning & $3.89(0.94)$ & $3.21(0.83)$ \\
13. Collaboration and medical activity & $3.38(0.92)$ & $2.84(0.88)$ \\
14. Self-development and -assessment & $3.85(0.82)$ & $3.21(0.83)$ \\
15. Leadership & $3.62(0.83)$ & $2.95(0.89)$ \\
\hline
\end{tabular}

SD: standard deviation.

Table 4. Importance Scores of Comparison Outcomes by Profession

\begin{tabular}{lcccccc}
\hline \multicolumn{1}{c}{ Outcomes } & $\begin{array}{c}\text { Private } \\
\text { practice }\end{array}$ & $\begin{array}{c}\text { General } \\
\text { hospital }\end{array}$ & $\begin{array}{c}\text { University } \\
\text { hospital }\end{array}$ & $\begin{array}{c}\text { House } \\
\text { staff }\end{array}$ & Others & p-value \\
\cline { 2 - 6 } & Mean (SD) & Mean (SD) & Mean (SD) & Mean (SD) & Mean (SD) & Kruskal Wallis \\
\hline 1. Medical knowledge \& clinical skills & $4.4(0.8)$ & $4.4(0.5)$ & $4.4(0.8)$ & $4.1(0.9)$ & $5.0(0.0)$ & 0.100 \\
2. Research & $3.5(1.0)$ & $3.3(1.0)$ & $3.4(0.9)$ & $3.6(0.9)$ & $3.5(1.3)$ & 0.800 \\
3. Solving problems & $3.8(0.9)$ & $4.3(0.7)$ & $3.7(0.8)$ & $3.5(0.9)$ & $3.8(1.0)$ & 0.100 \\
4. Foreign languages & $3.7(1.0)$ & $3.7(0.5)$ & $4.0(0.8)$ & $3.8(1.0)$ & $3.5(1.3)$ & 0.800 \\
5. Accountability & $4.3(0.6)$ & $4.2(0.8)$ & $4.1(0.8)$ & $4.1(0.7)$ & $4.3(1.0)$ & 1.000 \\
6. Knowledge of contemporary issues & $3.9(0.8)$ & $3.8(0.4)$ & $3.5(1.0)$ & $3.8(0.8)$ & $2.8(1.0)$ & 0.200 \\
7. Ethics \& morals & $4.3(0.8)$ & $4.1(0.6)$ & $4.2(0.9)$ & $4.0(0.7)$ & $3.8(0.5)$ & 0.300 \\
8. Teamwork & $4.1(0.9)$ & $3.9(0.6)$ & $4.0(0.8)$ & $4.0(0.7)$ & $4.0(0.8)$ & 0.900 \\
9. Keep a health body \& mind & $4.5(0.8)$ & $3.9(0.8)$ & $4.1(1.0)$ & $3.9(1.0)$ & $4.0(1.4)$ & 0.400 \\
10. Communication & $4.4(0.7)$ & $4.2(0.8)$ & $4.4(1.0)$ & $4.3(0.7)$ & $4.3(0.5)$ & 0.924 \\
11. Information management & $3.9(0.7)$ & $3.9(0.8)$ & $3.3(0.9)$ & $3.7(0.8)$ & $2.8(1.0)$ & 0.087 \\
12. Life-long learning & $4.2(1.0)$ & $4.1(0.8)$ & $4.0(0.9)$ & $3.8(0.9)$ & $3.0(0.8)$ & 0.183 \\
13. Collaboration \& medical activity & $3.5(0.8)$ & $3.2(0.7)$ & $3.3(1.1)$ & $3.5(0.9)$ & $3.0(1.4)$ & 0.655 \\
14. Self-development \& assessment & $4.1(0.7)$ & $3.8(0.8)$ & $3.6(0.9)$ & $3.9(0.8)$ & $4.5(0.6)$ & 0.299 \\
15. Leadership & $3.8(0.8)$ & $3.6(0.7)$ & $3.6(0.9)$ & $3.7(0.8)$ & $3.0(0.8)$ & 0.577 \\
Average & 4.02 & 3.90 & 3.83 & 3.84 & 3.67 & 0.488 \\
\hline
\end{tabular}

SD: standard deviation. 
(3.62) 등으로 나타났다.

반면 현재 자신의 능력 수준을 조사한 결과를 보면, 4점대의 학습성과는 한 개도 없었다. '외국어 능력'(2.76), '정보수 집'(2.79), ‘문제해결력'(2.80), ‘의학활동참여 능력'(2.84), ‘리 더십'(2.95), '의학연구 수행 능력'(2.95) 등의 학습성과는 2점 대로 상대적으로 낮은 편이었다.

\section{2. 직업에 따른 학습성과 중요도의 인식 차이}

Table 4는 직업에 따른 학습성과 중요도의 인식 정도를 나 타낸 것이다. 개원의, 봉직의, 교원, 전공의 등의 직업 중에서 개원의가 학습성과 전체 평균 4.02로 가장 높았다. 개원의는 '건강한 정신과 신체유지'(4.5)를 가장 중요하다고 인식하였으 며, '일차 의료수준의 의학적 지식과 술기'(4.4), '환자와 환자 가족과 효율적으로 의사소통하는 능력'(4.4), '의사로서의 사 회적 책임감'(4.3), '올바른 의학윤리'(4.3) 순으로 대답하였다. 봉직의는 '일차 의료수준의 의학적 지식과 술기'(4.4)를 가 장 중요한 학습성과로 인식하고 있었으며, '보건의료 문제 파 악 능력'(4.3), '환자와 환자 가족과 효율적으로 의사소통하는 능력'(4.2), '의사로서의 사회적 책임감'(4.2) 등의 학습성과를 중요하다고 대답하였다.

대학교원은 가장 중요한 학습성과로 '일차 의료수준의 의학 적 지식과 술기’(4.4)와 ‘환자와 환자 가족과 효율적으로 의사 소통하는 능력'(4.4)을 꼽았으며, '올바른 의학윤리'(4.3), '의 사로서의 사회적 책임감'(4.1), '건강한 정신과 신체유지'(4.1) 순으로 중요도가 나타났다.

전공의는 '환자와 환자 가족과 효율적으로 의사소통하는 능 력'(4.3)을 가장 중요한 학습성과로 대답하였으며, '일차 의료 수준의 의학적 지식과 술기'(4.1), ‘의사로서의 사회적 책임 감'(4.1)순으로 대답하였다. 군복무 및 제약회사 직원 등 기타 의 경우에는 응답자 모두(4명) '일차 의료수준의 의학적 지식 과 술기'(5.0)를 가장 중요한 학습성과라고 대답하였으며, '자 기개발 및 평가'(4.5)와 '의사로서의 사회적 책임감'(4.3) 순으 로 그 중요성을 인식하였다.

결과적으로 '일차 의료수준의 의학적 지식과 술기', '환자와 환자 가족과 효율적으로 의사소통하는 능력'과 '의사로서의 사회적 책임감 등의 학습성과는 모든 직업군에서 중요한 학 습성과로 공통적으로 나타났다. 반면 직업군에 따라 학습성
Table 5. Ranking and Score of Educational Needs

\begin{tabular}{lcc}
\hline \multicolumn{1}{c}{ Outcomes } & $\begin{array}{c}\text { Educational } \\
\text { needs score }\end{array}$ & Ranking \\
\hline 1. Medical knowledge and clinical skills & 3.14 & 3 \\
2. Research & 1.66 & 13 \\
3. Solving problems & 2.98 & 4 \\
4. Foreign languages & 3.56 & 2 \\
5. Accountability & 2.77 & 6 \\
6. Knowledge of contemporary issues & 1.83 & 11 \\
7. Ethics and morals & 1.81 & 12 \\
8. Teamwork & 1.54 & 15 \\
9. Keep a health body and mind & 2.78 & 5 \\
10. Communication & 3.99 & 1 \\
11. Information management & 2.65 & 7 \\
12. Life-long learning & 2.36 & 8 \\
13. Collaboration and medical activity & 1.66 & 13 \\
14. Self-development and assessment & 2.23 & 9 \\
15. Leadership & 2.21 & 10 \\
\hline
\end{tabular}

과에 대한 중요성의 우선 순위에 차이가 있었는데, 개원의는 '건강한 정신과 신체유지', '평생학습, 봉직의는 '문제해결력' 교원은 ‘올바른 의학윤리', 전공의는 '동료와의 협동'을 가장 중요한 학습성과로 인식하고 있는 것으로 나타났다.

\section{3. 교육 요구도 점수 및 교육 요구도 순위}

Table 5는 앞에서 분석한 중요도 인식과 현재 자신의 능력 수준의 차이를 이용하여 교육 요구도 점수와 순위를 나타낸 것이다. 15 개의 학습성과 중에서 교육 요구도가 가장 높은 학 습성과는 '환자와 환자 가족과 효율적으로 의사소통하는 능력' 이었으며, 다음으로 '외국어 능력'과 '일차 의료수준의 의학적 지식과 술기' 순으로 나타났다. 반면, '동료와의 협동정신', '의 학연구 능력', '의학활동 참여 능력'과 '올바른 의학윤리' 등은 상대적으로 낮은 교육 요구도를 보였다.

\section{고찰}

최근 주요 선진국에서는 대학의 경쟁력을 높이기 위한 국 가적 개혁 노력에 박차를 가하고 있다[14]. 1999년 볼로냐 선 언(Bologna declaration) 이후 많은 국가들은 고등교육혁신 
과 인적 자원의 경쟁력 강화, 평가인증, 고등교육시스템의 표 준화, 품질보증 등의 체제를 구축함으로써 대학의 경쟁력을 높이고자 노력하고 있다. 의학교육도 이러한 변화에 발맞추 고 있다. 의학교육의 질 향상(quality improvement) 노력은 세계의학교육연합(World Federation on Medical Education, WFME), 미국의 ACGME, 영국의 GMC, 유럽의 의학 교육학회(Association for Medical Education in Europe, $\mathrm{AMEE}$ ) 등에서 새로운 경향으로 정착되고 있고, 특히 유럽을 중심으로 '학습성과를 바탕으로 한 의학교육은 새로운 흐름 의 핵심으로 자리매김하고 있다[15]. 그러나 국내에서는 아직 까지 학습성과를 바탕으로 한 의학교육에 대한 소개가 드물 어 실질적인 의미의 교육이 시행되지 못하고 있는 형편이다. 본 연구는 아주대학교 의과대학 졸업생들을 대상으로 설문 조사를 실시하여 '의과대학 졸업생이 구비해야 하는 자질과 능력'이 무엇이며, '의과대학 학생들이 어떠한 능력을 함양하 기를 원하는지'에 대한 답을 찾고자 하였다. 물론, 국가마다 우선시 하는 졸업생들의 능력은 다를 수 있으며, 의학교육을 통해 어떤 능력을 길러야 할 것인가 하는 것은 재학생, 교수, 졸업생, 학부모, 고용주 등 사회적 합의를 요청하는 방대한 작 업이라고 할 수 있다. 본 연구가 일개 대상의 한정된 졸업생을 대상으로 하였다는 데에 한계점이 있지만 국내 의과대학에서 졸업생을 대상으로 그들이 말하는 학습성과와 교육 요구도를 파악한 것은 의미가 있다고 본다. 왜냐하면 사회가 요구하는 의사의 자질과 능력이 무엇인지, 그리고 이러한 자질과 능력 을 의과대학 재학 시 얼마나 잘 교육받았는지를 평가하기 위 해서는 그 프로그램을 성숙한 시각으로 바라볼 수 있는 졸업 생들의 의견이 중요한데 지금까지 국내에서는 이러한 작업이 제대로 이루어지지 않고 있었기 때문이다.

본 연구는 일개 의과대학 졸업생을 대상으로 연구자가 설 정한 15 개의 학습성과에 대한 중요도와 교육요구도 인식 정 도를 조사하고 의과대학 졸업생의 직업에 따라 학습성과 중 요도의 차이가 있는지 등을 알아보았다. 설문에 응한 졸업생 들은 15 개의 학습성과 중 ‘의사소통 능력, '의학적 지식과 술 기', ‘책임감, ‘의학윤리' 등의 학습성과를 중요한 것으로 응답 하였으며, 졸업생 모두 학습성과의 중요도에 비해 자신이 학 습성과를 습득한 현재 수준이 상대적으로 낮다고 인식하고 있었다. 특히, '외국어 능력', '의사소통 능력, '정보수집 능력'
등은 학습성과의 중요성과 자신의 현재 수준 간의 차이가 상 대적으로 큰 것으로 나타났다. 직업에 따른 학습성과의 중요 도 인식의 차이를 알아본 결과 직업군에 따라 최우선으로 강 조하고 있는 학습성과에 대해서는 약간의 차이가 나타났는데, 개원의는 '건강한 정신과 신체유지', 봉직의는 '문제해결력' 교원은 ‘의학윤리', 전공의는 ‘동료와의 협동심'이 그것이었다. 그러나 '일차 의료수준의 의학적 지식과 술기', '의사소통능력' '책임감' 등의 학습성과는 직업군과 상관없이 공통적으로 가 장 중요한 학습성과로 나타났으며 동시에 교육요구도 점수가 높게 나타났다. 이러한 결과는 좋은 의사의 자질을 규명한 Lee \& Ahn [12]의 연구 결과와 크게 다르지 않았다.

미국의 브라운 의과대학은 9 개의 능력, 즉 1) 효과적인 의 사소통, 2) 기본 임상술기, 3)과학적 진료에서 기초과학의 이 용, 4) 진단, 처치, 예방, 5) 평생학습, 6) 전문직업성 개발과 개인적 성장, 7) 보건 의료의 사회 및 지역사회적 맥락, 8) 도 덕적 추론과 임상윤리, 9) 문제해결 등을 학습성과로 제시하 고 있으며, 미국의 졸업 후 의학교육 인정평가기구인 $\mathrm{ACGME}$ 는 학습성과를 보다 간단하게 1) 환자진료, 2) 의학 지식, 3) 진료바탕학습과 향상, 4) 대인관계와 의사소통 기술, 5) 전문직업성, 6) 시스템바탕 진료 등 6개의 능력으로 기술 하였다[8]. 국내 의과대학의 경우에는, 서울의대는 학습성과 로 1) 의학의 이해와 적용, 2) 창의력, 3) 진료능력, 4) 의사소 통능력, 5) 문제해결능력, 6) 전문직업성, 7) 리더십 등 7개의 능력을 제시하고 있으며, 인제의대는 1) 진료능력, 2) 의사소 통능력, 3) 임상진료에서 과학적 지식 활용, 4) 문제해결력 및 비판적 사고, 5) 과학적 방법의 이해와 활용, 6) 자기주도적 평생학습, 7) 전문직업적 태도, 8) 리더십 등 8개의 능력을 졸 업 역량으로 선정하고 있다[16]. 브라운 의과대학, $\mathrm{ACGME}$ 의 전공의 평가 기준, 서울의대 학습성과, 인제의대 졸업역량 등 이 제시하고 있는 능력과 본 연구가 선정한 15 개 학습성과의 중요도가 매우 유사하게 평가된 것은 국가적 차이나 전공분 야의 차이에 상관없이 사회에서 요구하는 좋은 의사의 자질 과 능력이 비슷하다는 것을 보여준다.

그러나 본 연구가 제시한 15 개의 학습성과는 기존의 학습 성과와 차별화 되는 점이 있는데, 첫째, 윤리의식, 책임의식, 의사소통, 협동심, 리더십, 평생교육 등 '전문직업성'의 개념을 좀 더 구체적으로 제시하고 있다는 것과 둘째는, 건강과 외국 
어 능력 등을 강조하고 있다는 것이 그것이다. 이러한 차별성 은 아마도 연구자가 연구에 활용한 의과대학 교육목표의 성 격에서 비롯되었다고 보는데, 다시 말하면 학생들에게 기대 되는 행동을 구체적으로 표현해야 하는 교육목표의 기술상의 표현 때문에 발생한 것으로 해석된다.

본 연구의 교육요구도 조사 결과 졸업생들은 전문직업성의 요구가 대체로 높았다. 이러한 결과는 학습성과 중요도에 비 해 자신의 성취도가 낮다는 것을 의미하며 나아가 졸업생들 의 의견을 반영한 의과대학 교육과정개발이 고려되어야 함을 시사한다. 국내 의학교육에서는 10 여 년 전부터 전문직업성 에 대한 중요성을 인식하고 각 대학들이 전문직업성 함양을 교육목표로 설정하고 인문사회의학에 관련된 교과목들을 일 정 시간 교육과정에 반영하고 있음에도 불구하고, 본 연구 결 과와 같이, 전문직업성에 대한 학습성과의 졸업생의 교육적 요구가 높게 나타나고 있다는 점은 추후 연구 주제가 될 수 있다.

본 연구는 의과대학 교육기관의 비전과 미션이 반영된 교 육목표를 바탕으로 학습성과를 추출하여 교육목표가 구체화 되고 교육과정의 나침반 역할을 하는데 도움이 되고자 하였 다. 그러나 졸업 당시 학생들이 보여야 할 15 개의 학습성과는 보다 구체적으로 세분화되어 실제로 관찰하고 측정 가능한 행동용어로 표현할 수 있는 수준으로 다시 규명될 필요가 있 다. 학습성과를 달성할 수 있도록 기준과 수준을 결정하고 그 평가방법을 제시하는 등 교육과정을 설계할 수 있도록 하는 작업[10]은 연구자의 후속 과제이다. 정량적으로 입증하기 쉽 지 않은 윤리의식, 책임의식, 의사소통, 협동심, 리더십, 평생 교육, 시사적 지식, 외국어 능력 등의 학습성과 달성을 어떻게 증명할 것인가에 대한 문제는 계속적으로 연구되어야 할 것 이다[17,18].

전 세계 의과대학이 학습성과바탕교육이라는 개념을 점차 받아들이고 있으며 국제적인 의학교육기구들 또한 이 원칙을 지지하고 있다. 학습성과바탕교육은 다양화와 특성화를 존중 하고 자율성을 인정하는 대신 결과에 대한 책임을 묻는 성숙 한 방법론이라고 말할 수 있다. 현재 국내 의학교육 단계에서 학습성과바탕교육을 도입한다는 것은 그 자체가 커다란 부담 이 될 있다. 국내에서 학습성과바탕교육이 한 때의 유행으로 끝날지라도 학습성과에 대한 관심이 의학교육의 개인적·사회
적 적합성에 대한 지속적인 고민을 불러일으키는 것이라면 그 나름의 교육적 의미를 지난다고 할 수 있을 것이다. 이 점 에서 향후 학습성과바탕교육이 의학교육에 적용될 수 있도록 심층적인 연구가 요구된다.

\section{ACKNOWLEDGMENTS}

This work was supported by a National Research Foundation of Korea (NRF) grant, funded by the Korean government (MEST) (No. 2009-0064456).

\section{REFERENCES}

1. So KH. 'Competency' in the context of schooling: it's meaning and curricular implications. J Curriculum Stud 2007; 25: 1-21.

2. Shon MH. Competence, and everydayness of practical knowledge as its practical foundation. J Curriculum Stud 2006; 24: 1-25.

3. Caligiuri P, Santo VD. Global competence: what is it and can it be developed through global assignments? Hum Resour Plan 2001; 24: 27-35.

4. Davis MH, Amin Z, Grande JP, O'Neill AE, Pawlina W, Viggiano TR, Zuberi R. Case studies in outcome-based education. Med Teach 2007; 29: 717-722.

5. Smith SR, Dollase RH, Boss JA. Assessing students' performance in a competency-based curriculum. Acad Med 2003; 78: 97-107.

6. Schwarz MR, Wojtczak A. Global minimum essential requirements: a road towards competence-oriented medical education. Med Teach 2002; 24: 125-129.

7. Harden RM, Crosby JR, Davis MH. An introduction to outcome-based education. Med Teach 1999; 21: 7-14.

8. Harden RM. Learning outcomes and instructional objectives: is there a difference? Med Teach 2002; 24: 151- 
155.

9. Chae SJ, Lee YN, Kim HS. How to measure program outcomes? Proceedings of the Korean Society for Engineering Education Conference; 2005 Oct 12; Korean Society for Engineering Education; 2005. p. 64-74.

10. Dent JA, Harden RM. A practical guide for medical teachers. Edinburgh, UK: Churchill Livingstone; 2001.

11. Royal College of Physicians and Surgeons of Canada. Skills for the new millennium: report of the societal needs working group CanMEDS 2000 Project. Canadian medical education directions for specialists 2000 project. Ann RSPSC 1996; 29: 206-261.

12. Lee YM, Ahn DS. A preliminary study for exploring the attributes of being a good doctor. Korean J Med Educ 2007; 19: 313-323.

13. Kim JS, Choi YH, Kim SK. Analysis of in-service status and educational needs of engineering faculties. J Eng
Educ Res 2008; 11: 50-64.

14. Seo JH. A study on strategies of higher education for international competitiveness. High Educ 2003; 3: 101118.

15. Harden RM. Outcome-based education: the future is today. Med Teach 2007; 29: 625-629.

16. Lee BD. Outcomes based medical education. Proceeding of Workshop on Integrated Education at Ajou University School of Medicine; 2008 May 22; Ajou University School of Medicine; 2008. p. 1-29.

17. Whitcomb ME. Competency-based graduate medical education? Of course! But how should competency be assessed? Acad Med 2002; 77: 359-360.

18. Albanese MA, Mejicano G, Mullan P, Kokotailo P, Gruppen L. Defining characteristics of educational competencies. Med Educ 2008; 42: 248-255. 